\title{
Pneumologen-RLV gilt auch für Lungenärzte
}

\author{
Eine ausgelaufene Tätigkeitsbezeichnung schützt Ärzte nicht davor, beim Honorar unter die RLV- \\ Regelung zu fallen. Das hat das Bundessozialgericht im Fall eines Lungenarztes nun klargestellt.
}

Lungenärzte unterliegen den Regelleistungsvolumina (RLV) wie fachärztliche Internisten mit dem Schwerpunkt Pneumologie. Wie der Vertragsarztsenat des Bundessozialgerichts (BSG) in Kassel entschied, gilt für ausgelaufene Arztbezeichnungen generell die Zuordnung der heutigen Nachfolger. Mit dem Urteil billigte das Bundessozialgericht zudem erstmals die Übergangsregelungen zu den RLV 2005 in Bayern.

Konkret wiesen die Kasseler Richter einen Lungenarzt aus Bayern ab - einen Internisten mit der Teilgebietsbezeichnung „Lungen- und Bronchialheilkunde“. Im Streitquartal II/2005 hatte die Kassenärztliche Vereinigung (KV) einen gemeinsamen Honorartopf mit arztgruppenspezifischen Fallpunktzahlen für die fachärztlichen Internisten gebildet. Hier bezog die KV auch den Lungenarzt ein.

\section{Es kommt auf die Deutung an}

Der Arzt meinte, dies sei rechtswidrig. Denn der Bewertungsausschuss habe - anders als für fachärztliche Internisten - für die Arztgruppe der Lungenärzte gerade keine Regelleistungsvolumina vorgesehen. Während Widerspruch und Klage vor dem Sozialgericht München erfolglos blieben, gab das Bayerische Landessozialgericht in München dem Arzt recht.

Das BSG hob dieses Urteil nun jedoch auf und wies die Klage ab. Dass der Bewertungsausschuss Lungenärzte in seinem Beschluss zu den RLV nicht explizit als Arztgruppe genannt hat, stehe ihrer Einbeziehung nicht entgegen. „Der Umstand, dass der Bewertungsausschuss die den Lungenärzten vergleichbare Gruppe der fachärztlichen Internisten mit dem Schwerpunkt Pneumologie den RLV unterworfen hat, zeigt, dass die Nichtnennung der Lungenärzte allein darauf zurückzuführen ist, dass diese Arztbezeichnung seit 1988 nicht mehr erworben werden kann", begründeten die Kasseler Richter ihr Urteil.

Dabei betonte der BSG-Vertragsarztsenat, dass dies nicht nur für die Lungenärzte gilt. Auch andere Arztgruppen mit „ausge-

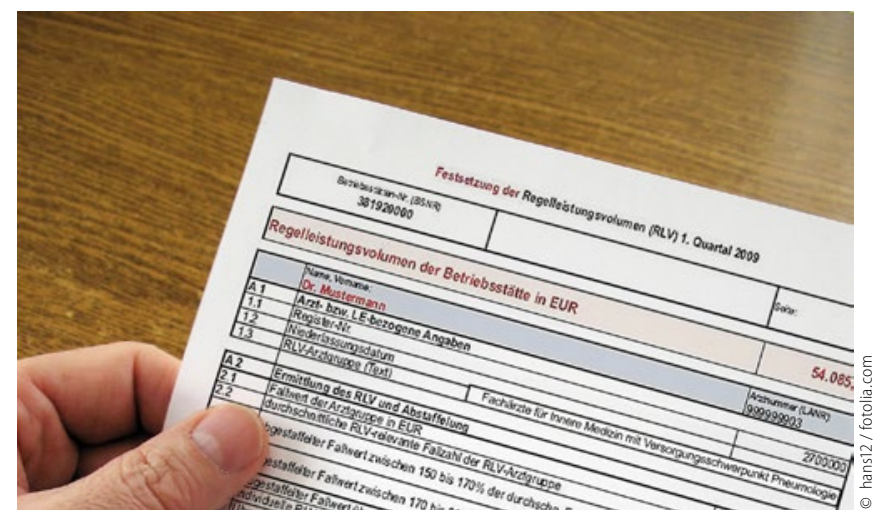

Lungenärzte unterliegen der RLV-Regelung wie fachärztliche Internisten mit dem Schwerpunkt Pneumologie. laufener" Gebietsbezeichnung seien „grundsätzlich der entsprechenden Arztgruppe nach dem heute geltenden Weiterbildungsrechts zuzuordnen".

\section{BSG bestätigt RLV in Bayern}

Mit seinem Urteil bestätigte das BSG zudem erstmals die Übergangsregelungen der KV Bayerns zur Einführung der RLV 2005. Die Honorarverteilung habe „maßgeblich auf arztgruppenspezifischen Fallpunktzahlen" beruht. Dass es hierbei zunächst keinen festen Punktwert gab, sei übergangsweise noch rechtmäßig gewesen. Der Zweck des festen Punktwerts sei es gewesen, das vertragsärztliche Honorar kalkulierbar zu machen.

Die Regelungen in Bayern hätten zu einem „zumindest annähernd festen Punktwert" geführt. In Verbindung mit den klaren Vorgaben für die Fallzahlzuwachsbegrenzung sei das Honorar "ausreichen kalkulierbar" gewesen, befand das BSG.

Martin Wortman

\section{Bewertungsportale: Patienten setzen auf Kompetenz}

Patienten entscheiden sich auf einem Arztbewertungsportal in der Regel für Mediziner mit den höchsten Wertungen in den Kategorien „kompetenter Eindruck" und "genommene Zeit". Nach dem Besuch eines Portals folgt auch oft der zeitnahe Besuch in der realen Praxis, die dem Patienten aufgrund des Profils gefällt. Das zeigt der 5. E-Patient Survey, dessen Ergebnisse das Portal jameda.de - bezogen auf die Antworten seiner Nutzer - im Juli veröffentlicht hat.

Für die Studie wurden über alle Portale hinweg 9.090 Nutzer befragt. Für die spezifische Auswertung hat jameda nach eigenen Angaben 1.147 seiner Portalnutzer gewinnen können. 87 \% nannten als wichtigstes Arztwahlkriterium den kompetenten Eindruck, der ihnen auf dem jeweiligen Arztprofil vermittelt wurde. Die guten Noten, die der Arzt in der Kategorie "genommene Zeit" erhalten hat, waren für rund $82 \%$ entscheidend. Auch Freitextkommentare (75 \%) spielten eine große Rolle: Sie geben oft Einblick in das Geschehen vor Ort, bzw. über den Grund, für die Zufriedenheit oder Unzufriedenheit des Bewerters.

Bei der Arztwahl spielte die Lage der Praxis für die Umfrageteilnehmer nur eine untergeordnete Rolle. Für mehr als $20 \%$ ist eine gute Verkehrsanbindung nicht wichtig. Das legt nahe, dass Patienten bereit sind, gröBeren Aufwand für eine ihrer Meinung nach gute Behandlung in Kauf zu nehmen. Etwa $18 \%$ legten bei ihrer Auswahl wenig Wert auf spezielle Leistungen des Arztes.

Interessant für Praxen ist, dass $77 \%$ der Nutzer aller Arztbewertungsportale laut Studie dieser Aussage zustimmten: „Nach dem Besuch des Arztbewertungsportals bin ich zum Arzt gegangen. Durch die Meinung/ Bewertung anderer Patienten auf dem Bewertungsportal habe ich den für mich passenden Arzt gefunden." Nach Lesart von jameda zeigt dies, wie etabliert die Portale bereits darin sind, Patienten Orientierung bei der Arztwahl zu geben. 